\title{
Los estudios legislativos. ¿Dónde estamos? ${ }^{\text {¿ }}$
}

Legislative studies. Where are we?

\author{
Elia Edith Argüelles Barrientos ${ }^{2}$ \\ Mulio César Contreras Manrique ${ }^{3}$
}

Fecha de recepción: 5 de mayo de 2014

Fecha de aprobación: 9 septiembre de 2014

\section{RESUMEN}

El objetivo primordial del articulo es compartir reflexiones en torno a los estudios del poder Legislativos realizados en México y cuáles son sus retos, en este sentido el Congreso se observa como una institución política que ha cobrado relevancia en el país para los estudiosos de la política a través de un proceso gradual y ante una sociedad con un sistema político en movimiento que requiere de seguir aumentando el interés en este eje temático.

Palabras clave: instituciones política, poder Legislativo, Ciencia Política.

\section{Abstract}

The primary objective of this article is to share thoughts reflections on studies of the Legislative power made in Mexico and what are its challenges, and in this regard the Congress is seen as a political institution that has gained relevance in the country for the academics of politics through

1 El articulo es producto del proyecto de investigación institucional del Colegio de San Luis, A.C., denominado : "Los Estudios Legislativos"; y es financiado por la referida Institución.

2 Magister en Asuntos políticos y políticas públicas, Colegio de San Luis A. C. Estudiante Doctorado en Ciencias Sociales, Colegio de San Luis, A. C. Correo Electrónico: edith_arguelles@hotmail.com

3 Doctor en ciencia política, Universidad Autónoma Metropolitana. Profesor-Investigador, Programa de Estudios Políticos e Internacionales - Colegio de San Luis. A. C. Correo Electrónico: ccontreras@colsan.edu.mx 
a gradual process and before a society with a political system in motion which requires to continue increasing the interest in this theme

Keywords: Political institutions, legislative power, Political Science

\section{INTRODUCCIÓN}

El poder Legislativo es una de las instituciones políticas más representativas dentro de un Estado Democrático, su papel es preponderante para el funcionamiento del sistema de pesos y contrapesos, equilibrio que evita que el poder dentro de un sistema político se auto-regule como en los Estados absolutistas, tiranos o dictadores.

Posterior al proceso revolucionario ${ }^{4}$ y la conformación hegemónica de cierto grupo al poder ${ }^{5}$ en México el rol del Legislativo en apariencia se mantuvo estático pormucho tiempo fungiendo, con el papel de un sellador de estampas al aprobar sin cuestionamientos las iniciativas del ejecutivo.

No fue hasta 1997 cuando por primera vez el Partido Revolucionario Institucional (PRI) perdió la mayoría absoluta en la Cámara de Diputados, que los estudiosos de la Ciencia Política comenzaron a interesarse en observar el nuevo rol que esta institución emprendió en el contexto de un sistema político pluralista donde

4 Para Camacho Vargas (2013 [2006], p. 106) : “El triunfo de la Revolución constitucionalista propiciaba las condiciones adecuadas para que el 19 de septiembre de 1916 se convocara a la integración de un nuevo Congreso Constituyente, a fin de dotar al país de un nuevo ordenamiento político, jurídico, económico y social, mediante reformas a la Constitución de 1857 "

5 Desde 1928 cuando el presidente Plutarco Elías Calles propuso la creación del Partido Nacional Revolucionario (PNR), conformándose el partido un ańo después el grupo vencedor de la revolución mexicana mantuvo su hegemonía en los gobiernos federal y en los 32 estados hasta 1989. Nueve años después, en 1938, luego de la ruptura entre Calles y el entonces presidente Lázaro Cárdenas, en la cual participaron varios miembros distinguidos del partido, como el ex presidente Emilio Portes Gil, se realizó un cambio en las directivas del partido a nivel nacional, y en sus filas se incluyó a varias centrales obreras del país que hasta entonces estaban oficialmente fuera del partido y se cambió el nombre de la institución por el de Partido de la Revolución Mexicana (PRM). En 1946, el nombre tomó giro dando vida al Partido Revolucionario Institucional que hasta el día de hoy permanece. 
diferentes organizaciones políticas comenzaron a disputarse y se disputan al día de hoy el control político en el país.

En ese sentido, la presente trabajo ofrece un breve análisis de la revisión teórica respecto de los estudios realizados en México en torno al poder Legislativo como un primer acercamiento dentro de un proyecto de investigación legislativa más amplio.

El trabajo que aquí se expone se desarrolla como parte del fundamento teórico del proyecto de tesis doctoral en curso denominado Instituciones politicas y politicas públicas: el rol del congreso local en la construcción de la politica de seguridad en San Luis Potosi (2006-2015), dentro del Programa del Ciencias Sociales con énfasis disciplinar en Ciencia Política que se ofrece en el Colegio de San Luis A.C. (Colsan), centro de investigación que forma parte de la red del Consejo Nacional de Ciencia y Tecnología (Conacyt) en México.

El objetivo de este trabajo es mostrar una revisión de los campos en los que la Ciencia Política en México ha transitado dentro de los estudios parlamentarios, esto apoyada de autores que previamente han realizado algunos balances en este sentido, dando cuenta que la disciplina ha caminado en este terreno en un proceso de transición política que se ha reflejado en esta área del conocimiento.

La propuesta se presenta en tres apartados, en un primer momento se mostrará una breve reseña histórica sobre la institucionalidad cambiante de Congreso Mexicano, posteriormente un recorrido sobre los estudios legislativos en México, un primer acercamiento a sus categorías, enfoques, metodologías, modelos, tópicos e intereses, para continuar con una sección respecto de aquellos pendientes que en la materia quedan aún por recorrer.

La intención es realizar un análisis de los alcances de los estudios legislativos, así como vislumbrar los nuevos retos ante un contexto de alternancia en las diferentes esferas políticas del país donde no existe seguridad plena respecto de quiénes ocuparán los espacios parlamentarios, dado la diversificación que se ha realizado en las votaciones de los ciudadanos. 
Finalmente se ofrecen algunas reflexiones en torno a los retos del campo de estudios legislativos en México y algunas propuestas para que otras instituciones académicas, cívicas y el propio poder Legislativo, puedan incentivar el desarrollo de los mismos.

\section{El Congreso en MéXico y su nueva institucionalidad}

Es consenso dentro de los estudios de la política en México, que la apertura del sistema sea un proceso lento pero constante que se ha consolidado en las últimas décadas, incluyendo así la creación y la consolidación de instituciones que coadyuven a la convivencia de una pluralidad de actores dentro del escenario político de México.

Para el caso del poder Legislativo dentro de la lógica de división de poderes la autonomía de esta organización se ha presentado en ciertos momentos clave que fueron posibles gracias a la conformación de reformas dentro de las reglas electorales que han dado cause al Estado Mexicano.

La pluralización del sistema democrático ha sido gradual; para el caso de la Cámara de Diputados comienzan a sentarse las bases en 1964, mientras que para los congresos locales comienza a partir de 1974 cuando se da paso a lo que se llama la construcción institucional pluralista. Mientras que al Senado de la República dicha apertura a través de la representación proporcional no llegaría sino hasta la reforma de 1993 que se presentó en el país.

A decir de Acosta (2009) fue una compleja dinámica de una oposición de derechas e izquierdas la que se fue modificando mediante negociaciones políticas, conflictos locales y reformas electorales, el rostro autoritario del régimen ${ }^{6}$ que existía en el país.

Si bien la Reforma Política de 1977 fue fundamental en la historia de la construcción del sistema de partidos (Lujambio, 2013 [2010]) que hoy se tiene, también lo fue para dar inicio a la llamada transición democrática. En ese año también "la Constitución obligó a las legislaturas estatales a aprobar sistemas electorales mixtos, es decir, a

6 Priísta. 
que incluyeran diputados de representación proporcional en su integración. Se trató de un paso histórico fundamental para la pluralización de nuestras instituciones legislativas”, (Lujambio 2013 [2010], p. 420).

Siguiendo la perspectiva de Lujambio (2013 [2010]), de este proceso se ha desprendido el hecho de que los congresos locales desaparecieron sus mayorías calificadas ${ }^{7}$; con lo que de un solo partido concretaron una dinámica hacia los gobiernos divididos que de esta forma fueron apareciendo en el contexto de las entidades del país.

Para Contreras y Rodríguez (2011: pp. 436-437) en lo que se refiere a los congresos locales durante la fase del partido hegemónico se entiende que éstos replicaban lo que acontecía antes de 1997 en el congreso federal, es decir, su "nulo protagonismo institucional y ausencia de una toma de decisiones plural, colegiada y autónoma”; mientras que -en contraste con otras posiciones- en algunos casos aún y alcanzándose la figura de gobiernos divididos en las entidades los congresos siguieron manteniéndose pasivos aún sin la mayoría calificada de un partido, debido a que no existía un posicionamiento de las fuerzas políticas que pudiesen generar un contrapeso con los demás poderes del Estado.

Sin embargo, no se puede negar que el resultado de este proceso de transición fue el fortalecimiento de los partidos políticos y de los sistemas de partidos, la posibilidad de una representación política en la cual sociedad mexicana vea reflejados sus intereses dentro de la acción legislativa, pero principalmente una nueva forma de institucionalidad en donde esta organización comienza a generar una dinámica de formas de debate más abierto entre las fuerzas del país y menos cerrado hacia los ciudadanos, sostiene Lujambio, (2013 [2010]).

Parte del debate de los estudiosos del tema es que la transición política en el poder Legislativo ha traído como consecuencia entre otras cosas que ni el poder ejecutivo ni su partido tengan garantizada la hegemonía en las decisiones que toman los diputados. Esto, a decir de Arrieta (2010)

7 Una mayoría calificada es cuando existe la presencia de dos terceras partes de los escaños en poder de un solo partido. 
ha generado una autonomía en la toma de decisiones por parte de los legisladores, que llevan a una dinámica desarrollada en el Congreso privilegia los acuerdos, lo que favorece a que se conforme una institución plural debido a que en él están representados la mayoría de los intereses sociales... (p.58)

De esta forma, "la relación entre el Ejecutivo y el Congreso se vio hondamente modificada luego de que el titular del gobierno federal dejó de contar con una mayoría dispuesta a apoyar sus proyectos y de que las restricciones constitucionales para llevar las riendas de este proceso se hicieron manifiestas”, (Bejar, 2012, p. 620).

Todo esto para los estudiosos del tema, llevó a la generación de una independencia del poder Legislativo respecto al Ejecutivo y a una lógica de la división de poderes más acorde a los principios del sistema de pesos y contrapesos. De esta manera se sentaron las bases para lo que Valencia (2010, p.195) llama una tendencia "hacia un sistema plural, dividido y con fuertes reticencias institucionales para la consolidación democrática”.

Por su parte, Acosta (2009) considera que estos ańos representaron el proceso de desestructuración del régimen político pos-revolucionario representado por el PRI, que simbolizaba tanto un sistema de partido virtualmente único como un "hiperpresidencialismo" sin igual en América Latina. Todo esto proceso que representa "el paso de un régimen político centralizado a uno descentralizado" abonó positivamente de acuerdo a Bejar (2012, p. 620) en un fortalecimiento de la representación política subnacional y sus liderazgos como producto del diseño federal constitucional, lo que trajo consigo un aumento en la competitividad electoral en buena parte del país.

En los albores del siglo XXI en nuestro país se consolidó la alternancia política en el Ejecutivo federal y el Congreso se convirtió en un órgano de representación nacional que arropó "las distintas sensibilidades políticas, adquiriendo una de las características de las democracias pluralistas" sostienen Toledo y Weldon (2007, p. 5).

Para los autores esta dinámica representó un arribo del pluralismo, que ha repercutido en un mayor involucramiento de los ciudadanos así como de sus expectativas ante la política; son personas más informadas con mayores expectativas de sus legisladores y 
que se involucran más en la vida política, lo que a su vez ha traído como consecuencia que el poder Legislativo tenga mayor diálogo y acuerdo entre grupos parlamentarios para su funcionamiento.

Aunque la cuestión de la representación política de estos grupos parlamentarios todavía está en análisis en tanto su acción de legislar de acuerdo a las verdaderas necesidades de los diferentes realidades de la sociedad.

Un fenómeno cambiante dentro de la manera de operar del legislativo, se presentó a partir del 2012 cuando el Partido Revolucionario Institucional regresó a ocupar el poder Ejecutivo después de dos sexenios. El proceso que se ha venido dando desde el retorno de la organización partidista a la presidencia es una serie de estrategias que han permitido que nuevamente sus iniciativas sean aceptadas por la mayor parte de las diversas fuerzas políticas dentro del país, aunque en un contexto de mayor debate que cuando sólo predominaba el Revolucionario Institucional en todas las esferas del poder, es por eso que el partido ha mostrado la maestría y experiencia que en el control de sistema político tuvo por muchos años.

\section{Los estudios del poder Legislativo en la Ciencia Política EN MÉXICO}

La transición del sistema político nacional representó para el mundo de la academia y en especial para la Ciencia Política un nicho de oportunidades para el estudio de las nuevas realidades en las esferas públicas; pues la realidad del contexto político ha permeado las temáticas de estudios en la materia y aspectos que no habían sido explorados comenzaron a ser agenda entre los estudiosos de la vida política.

El impacto que han tenido los cambios políticos en la disciplina son bien plasmados en un análisis realizado por Acosta (2009) sobre el comportamiento de los estudios de las trayectorias de las diversas "comunidades epistémicas" que configuraron el campo contemporáneo de la Ciencia Política en México entre los años 1980 a 2008. El autor sostiene que el análisis politológico mexicano de los últimos años gira en torno a tres momentos principales del cambio político los cuales podrían resumirse 
en tres momentos: Crisis del autoritarismo mexicano; Transición politica e Ineficacia institucional y problemas de consolidación democrática.

De acuerdo con Acosta (2009) en los treinta años que incluyen su estudio ${ }^{8}$ la actividad política se volvió el centro de atención de intelectuales y académicos de las Ciencias Sociales, así como de los medios de comunicación en México. Este proceso se convirtió en el objeto de estudio de nuevas generaciones de sociólogos y politólogos de muy diversas escuelas y corrientes teóricas, que conformaron agendas de investigación con una variedad de métodos, productos y orientaciones. El proceso de cambio político fue acompańado por la estructuración de una agenda de investigación y debate académico que articuló diversas "comunidades epistémicas" en torno a la Ciencia Política mexicana contemporánea.

El primero de los momentos que sugiere Acosta (2009) dentro de la disciplina es la Crisis del autoritarismo mexicano, período que trasciende en la década de los ochenta, en un contexto de crisis económica e incapacidad política para que el régimen hegemónico siguiese manteniéndose, los temas de estudio fueron el régimen político, comportamiento electoral, sistema de partidos, liderazgos políticos, rebeliones cívicas y crisis del corporativismo.

El segundo período denominado Transición politica y problemas de consolidación democrática que corresponde a la década de los 90's se vio envuelto de tópicos como alternancia política, el surgimiento de nuevas agrupaciones políticas, el análisis del desempeño gubernamental, la gobernabilidad y el cambio político.

La temática nombrada Ineficacia institucional y los problemas de consolidación democrática, se desarrolló una vez concretada la alternancia política en la Presidencia de la República, en un momento que el autor llamó la "reconfiguración de los equilibrios políticos en un régimen en transición". Los temas de estudio en este período fueron: gobiernos divididos, políticas públicas, gobernanza, disciplina partidista y comportamiento del legislativo.

8 Agregaríamos que este interés sigue en aumento al día de hoy. 
"Los tres ciclos permiten apreciar agendas, temas y métodos de investigación que combinaron acercamientos cuantitativos, cualitativos y mixtos en la exploración de los fenómenos políticos contemporáneos del país" (Acosta, 2009, p.103). Si bien el autor refleja en su clasificación los principales temas de estudio que ha abordado la Ciencia Política durante las últimas tres décadas, aquellos encaminados al estudio del poder Legislativo no predominaron en comparación con el resto de los tópicos de análisis, adicionalmente podemos agregar que la mayor presencia de análisis encaminados a observar el comportamiento legislativo se ubican una vez que inicia la alternancia política dentro del gobierno Federal en la década en que el presidente Vicente Fox tumbó al PRI de Los Pinos.

Aquellos intereses de observar el papel del poder Legislativo surgieron dentro del segundo y tercer momento que plantea Acosta (2009), cuando se presentó la transición política que abrió el sistema a una mayor competencia y competitividad dentro del país. Anteriormente la ausencia de estudios en este sentido, se puede explicar con lo que estudiosos en el ámbito como Valencia (2010, p.195) explican, fue una inercia bajo la cual estuvo sujeta la mayor parte del siglo XX en el terreno, misma que descartó "los análisis en torno al poder Legislativo mexicano y las reglas que de esta organización emanan, dado que para fines prácticos el presidente de la República era el único legislador”.

Si bien la inercia en el sistema hegemónico ocasionó que existiera una limitada producción de las tareas legislativas, por parte de los estudiosos de la política la ausencia de equilibrios y la total dependencia a la que estaban sujetos los legisladores vinculados al partido del régimen descartó la posibilidad de análisis exclusivos sobre el poder Legislativo, lo que produjo una serie de documentos en torno a la figura presidencial y al partido del régimen, dejando de lado la investigación sobre la actividad legislativa (Molinar y Weldon, 2009; Valencia, 2010).

Sin embargo, "la evolución histórica del sistema político mexicano ha empezado a enmendar los desequilibrios y a restituir el papel real del Congreso en la vida del sistema político mexicano", (Valencia, 2010, p.196); en ese sentido, ha sido posible el crecimiento de los estudios parlamentarios dado que el cambio de dinámica legislativa favoreció a que los estudiosos voltearán a registrar este proceso y más. 
En su recuento del desarrollo de los estudios congresionales en México, Lujambio (2013) reseńa que las primeras investigaciones en torno al poder Legislativo fueron desarrolladas por interesados en las Ciencias Jurídicas y el Derecho quienes describieron básicamente la producción legislativa, otro nicho de intereses en los estudios legislativos se encuentra en los historiadores quienes han realizado un trabajo descriptivo dibujando los diversos escenarios del Congreso Mexicano a lo largo de diferentes períodos históricos desde su conformación a la fecha; en tanto que la Ciencia Política es la última disciplina que volteó al análisis de esta institución, una vez que se presentó la apertura del sistema político.

Ya dentro del terreno de la disciplina que a este trabajo compete, Contreras y Rodríguez (2011, p. 435) sostienen que en las últimas décadas del siglo pasado y principios del presente, el poder Legislativo en México ha manifestado cambios significativos los cuales han pasado por las fases de una hegemonía partidista y de lo que llamarían Labastida y López, (2004, p. 749) una transición democrática prolongada marcadas por reformas y cambios políticos importantes.

Contreras y Rodríguez (2011) consideran que una prueba de que la mirada de los diversos actores nacionales y locales entre ellos los académicos se ha enfocado cada vez más al registro del activo papel que los legisladores han manifestado de los últimos años, está en la proliferación del análisis de las nuevas realidades tales como la pluralidad federal y los gobiernos divididos en las entidades.

En este sentido, los autores (2011, p. 436) realizan una recopilación de los temas que han predominado entre los estudiosos del poder Legislativo a nivel federal en los últimos años encontrando como objetos de estudio destacados las siguientes temáticas: la eficacia en el proceso legislativo (Mora, 2006); la representación democrática (Hurtado, 1999 y 2001); el actuar de las mayorías, las minorías y el trabajo en comisiones (Nacif, 2000, 2000a, 2002, 2003; Béjar, 2006; Valencia, 2007: Mora, 2007); la relación entre representación parlamentaria y disciplina partidista (Béjar, 2003; Jiménez-Ottalengo y Solórzano, 2007 Gimate-Welsh y Rodríguez, 2007); la actuación partidista (Meyenberg, 2003) y los efectos de la alternancia (Casar, 1999; Huber, 2003). 
Así también el desempeño legislativo (Bejar y Ugalde, 2003, Nacif y Sada, 2006; Díaz, 2006; Díaz, et al 2008.); el estado de la separación de poderes (Lujambio, 2003, Casar, 1998); el diseño institucional y las prácticas formales del proceso legislativo (Heller y Weldon, 2003; Sirvent, 2003; Loira 2007; Heller, 2007); los cambios institucionales (Bátiz, 1999; Rossell, 2002; Elizondo y Nacif 2003; Riva Palacio, 2007.); las relaciones Legislativo-Ejecutivo (Casar, 2002; Romero, 2007; Varela, 2007; Espinoza y Cárdenas, 2007), las relaciones Legislativo-Judicial (López, 2007.); negociación y cabildeo (Arrieta y González, 2007; Muñoz, 2007; Dworak, 2011.); reelección de diputados (Lujambio, 1993 y 1996; Weldon, Campos, Carega y Mejía, en Dworak 2003).

Para Contreras y Rodríguez (2011:437) los estudios sobre las entidades legislativas a nivel sub-nacional no se han hecho esperar; algunas de las cuestiones que mayormente destacan son los cambios institucionales y su fortalecimiento (Molina, 2004; Raigosa, 2004; Ortiz, 2005; Vega, 2009), fiscalización y rendición de cuentas (Guerrero, 2001; Pardinas, 2004; Pérez y Cejudo, 2005), materia presupuestal (Aziz, 2002), agendas y reformas legislativas (Escobedo, 2005), análisis entre las instituciones legislativas o derecho comparado (Valadez, 2005), equidad de género y Servicio Civil de Carrera (Orta y Nava, 2004), elites parlamentarias en los estados (Jiménez et al, 2007), relaciones Legislativo-Ejecutivo (Vega y Hernández, 2007), gobiernos sin mayoría y coaliciones (López, 2007), relaciones entre el jefe del Ejecutivo y su partido en el Legislativo (Mirón, 2007) y sistema de comisiones (Córdova, 2004).

De esta manera todo el estudio en la materia ha ido evolucionando de acuerdo a los propios movimientos y reorganizaciones de la vida parlamentaria como una institución política en donde su estructura así como sus funciones y acciones, giran en torno a dinámicas sociales no estáticas en donde la alternancia política se mantiene activa, pero las viejas prácticas de los tiempos hegemónicos siguen siendo un fantasma visibles dentro de los escenarios políticos del México actual.

\section{NUEVOS CAMPOS Y RETOS EN LOS ESTUDIOS LEGISLATIVOS}

Si bien los estudios legislativos han llegado para quedarse no sólo dentro de la Ciencia Política sino también dentro de otras disciplinas sociales, aún quedan nuevos 
campos que explorar, incluso muchos que sólo pueden explicarse bajo la lógica del contexto nacional alejados de los marcos teóricos explicativos anglosajones de donde provienen las raíces de los estudios parlamentarios.

En ese sentido, a continuación se exponen algunas ideas preliminares sobre cuáles son esos campos nuevos por explorar y aquellos retos en la investigación de los estudios legislativos. Uno de estos retos es sin duda, el estudio de los congresos locales, si bien cada vez la disciplina se expande en México, las investigaciones en torno a estas organizaciones políticas a nivel subnacional son todavía escasas.

Recordemos que los años noventa se identificaron como el período en el que el poder Legislativo comenzó a estudiarse en el plano subnacional (Reveles, 2011, p.11), fue momento en que los congresos locales llamaron la atención al seguir la lógica federal en donde su composición comenzó a ser diversa respecto a la titularidad de los poderes ejecutivos locales, es decir, se conformaron los gobiernos divididos dentro de éstos.

En una evaluación realizada por Reveles (2011) sobre los estudios relacionados con congresos locales respecto a temas tratados, enfoques teóricos y metodologías utilizadas, considera que "casi todo está por hacer" (p. 26). Se ha trascendido en temáticas como el marco legal, los gobiernos sin mayoría, las élites parlamentarias y lo relacionado con las cuestiones electorales, sin embargo el autor comparte la preocupación en torno a que es urgente analizar a fondo nuevas esferas.

Algunas de sus propuestas de análisis giran en torno al estudio de las funciones representativa, legislativa y de control, así como lo que implica cada una de éstas. Otros puntos que destaca el autor que son necesarios estudiar son las reformas integrales, la producción de leyes, la influencia del Legislativo en el nombramiento de gobernantes y en la elaboración o enmienda de políticas públicas, así como en la distribución y fiscalización de los recursos públicos.

Dentro de los nuevos campos de estudio por abordar, Contreras y Rodríguez (2011) sostienen que llama la atención dentro de la significativa y amplia gama de intereses surgidos desde los politólogos hacia el Congreso federal, que no existan antecedentes 
del abordaje de la institución política desde perspectivas como la organizacional. Lo que a decir de los autores, podría explicarse como una escasa relación de la Ciencia Política en México con otras disciplinas o campos de estudio de lo social, que sostienen sí se ha presentado por los estudiosos norteamericanos del poder Legislativo.

Contreras y Rodríguez (2011) en su trabajo exponen la pertinencia de estudiar al Congreso desde una perspectiva organizacional, dado que las características de las organizaciones que se encuentran en las legislaturas y toman de análisis la estructura organizacional de un caso representativo como lo es el Congreso del Estado de San Luis Potosí, utilizando como metodología la herramienta del diseño organizacional.

Su propuesta transita en la apuesta de que el congreso puede ser analizado dentro de los argumentos organizacionales, por lo que se convierte en un objeto de estudio del zoo organizacional. En ese sentido la propuesta es que las legislaturas pueden ser estudiadas desde la perspectiva del estudio de la ciencia política y el estudio de las organizaciones.

Retomando la relevancia de los congresos locales, es importante destacar que parte de los estudios legislativos se han concentrado en la figura del legislativo federal, principalmente en la Cámara de Diputados, siendo importante resaltar que el análisis y descripción de los Congresos locales ya no queden en un segundo plano.

Lo anterior responde a diversos aspectos por un lado se encuentra el factor educativo, si se toma en cuenta que los estudios de Ciencia Política tienen unas cuantas décadas en el país y por otro lado que en sus inicios la mayor parte de dichos programas se concentraban en la capital de éste.

La expansión de programas en la disciplina transcendió hacia las entidades de México en los años recientes, lo que ha permitido que el análisis de las legislaturas comience a ser de interés así como otro tipo de temáticas que poco o nada habían sido tocadas en relación con la política local desde un análisis científico politológico, habiendo sido anteriormente materia de otras disciplinas como el Derecho, Comunicación, Historia, las Relaciones Internacionales, entre otras. 
En este sentido aunque incipientes los trabajos que desde la academia se realizan en materia de los congresos locales corresponden a un primer acercamiento a reflejar la institucionalidad pasada y presente de estas entidades políticas, que puedan mostrar una prospectiva futura de las mismas, por lo que la temática representa un nicho de oportunidad para los estudiosos de la política.

Ahora bien, la propuesta es atreverse a vincular el estudio de los congresos locales con otras variables en este caso su relación en el proceso de formulación de políticas públicas, con su relación con los ejecutivos, con los municipios, relación con el congreso federal y otros congresos locales, con otros poderes dentro del propio estado, con la ciudadanía, pasando de un estudio mayormente descriptivo a uno que refleje la realidad en torno a cómo gira la dinámica de esta organización.

\section{Conclusiones}

Los anteriores fueron algunas reflexiones que a lo largo del texto se plasmaron respecto a cómo se han desarrollado los estudios legislativos en México y su importancia en la vida política del país. Sin duda dentro de esta propuesta quedan múltiples aristas por abarcar que son importantes dentro de los estudios congresionales; sin embargo la intención de estas consideraciones es realizar un llamado a los politólogos tanto nacionales como extranjeros a mirar a esta institución política como una buen objeto de estudio, sin tener miedo a proponer nuevos temas ni a vincularse con otras disciplinas para explorar estudios de interfase.

Las instituciones políticas siguen importando en el mundo de la Ciencia Política y otras disciplinas pues constrińnen la vida de los ciudadanos dentro de la sociedad inmersa dentro del Estado, para el caso de México no es la excepción se cuenta con una figura en constante movimiento, son evidentes los estudios en torno al congreso federal y su proceso hegemónico hacia su apertura, pero los estudios en los congresos locales hay mucho por explorar. 


\section{REFERENCIAS}

Acosta, A. (2009). La política en México: ideas, anteojos y cristales. En Andamios, Volumen 6, número 11, agosto, 2009, pp. 101-126.

Arrieta, L. (2010). La acción de los grupos de interés en la nueva institucionalidad del Congreso mexicano. Estudios políticos, núm. 20, Novena época, mayo-agosto, 2010, pp. 57-84

Béjar, L. (2012). ¿Quién legisla en México? Descentralización y proceso legislativo. Universidad Nacional Autónoma de México-Instituto de Investigaciones Sociales. Revista Mexicana de Sociología 74, núm. 4 (octubre-diciembre, 2012): 619-647. México, D.F. ISSN: 0188-2503/12/07404-04.

Contreras, J. y Rodríguez, E. (2011). "La institución legislativa como una organización. Consecuencias para su estudio.” En Raúl López Flores, Fermín Edgardo Rivas Prats, Armando Hernández Cruz, Alfredo Sainez Araiza (coordinadores) Estrategia y Práctica Parlamentaria en un Congreso plural. Senado de la República. LXI Legislatura. (C) Instituto Belisario Domínguez Primera edición, 2011.

Lujambio, A. (2010). "25 años de pluralización de los Congresos locales de México (19741999)” en Lujambio, Alonso (2010). Estudios congresionales, Ciudad de México, Ediciones Mesa Directiva Cámara de Diputados Legislatura, pp. 417-447

Reveles, F. (2011). Los estudios sobre los congresos locales en México. Temas y problemas. En Estudios políticos, núm. 23, Novena época, mayo-agosto, 2011, pp. 11-30.

Valencia, L. (2010). "La dinámica histórica del proceso de formación y consolidación del poder legislativo en México", en Ernesto Soto Reyes Garmendia (Coordinador) La democracia y el sistema político en México. Ciudad de México, Universidad Autónoma Metropolitana, pp 195-230. 